\title{
O exame de imagem do segmento anterior no diagnóstico de certeza da catarata branca intumescente
}

\section{Image test in the sure diagnosis of intumescent white cataract}

Virgilio Centurion'; Edson Branzoni Leal'; Augusto Cézar Lacava'

\section{Resumo}

Objetivo: Identificar aspectos na ACSA (analise computadorizada do segmento anterior) que levem ao diagnóstico de certeza de CBI (catarata branca intumescente). Métodos: Dois grupos de olhos, com catarata branca e nigra são estudados utilizando-se os critérios clínico e laboratorial. Resultados: A espessura do cristalino $\geq 5.36 \mathrm{~mm}$ e o seu aspecto esferiforme foram evidenciados como dados mais freqüentes na catarata branca intumescente (CBI). Conclusão: A utilização da ACSA fornece dados importantes no diagnóstico de certeza da CBI.

Descritores: Catarata/diagnóstico; Segmento anterior do olho; Processamento de imagem assistida por computador; Interpretação de imagem assistida por computador

Oftalmologistas do Instituto de Moléstias Oculares - IMO - São Paulo (SP), Brasil. 


\section{INTRODUÇÃO}

A catarata tem várias formas de classificação e esta poderá seguir o critério da sua etiologia, das características clínicas ou do seu grau de evolução ${ }^{(1)}$.

$\mathrm{Se}$, ao exame biomicroscópico do segmento anterior observarmos uma "mancha branca" na região pupilaré possível que estejamos perante um quadro de catarata branca ${ }^{(1.2)}$.

Esta catarata branca, por sua vez, devido a suas características pode ter várias formas clínicas, que devem ser corretamente diagnosticadas para que possamos indicar a estratégia cirúrgica apropriada ${ }^{(2-4)}$.

Em relação à etiologia, a catarata relacionada à idade tem como fatores determinantes a idade e o hábito de fumar. Outros possíveis fatores etiológicos seriam o excesso de radiação ultravioleta, fatores alimentares, trauma, infecção ou inflamação pós-cirurgia vítreo retiniana.

O objetivo do presente trabalho é demonstrar a importância do exame de imagem do segmento anterior por meio da análise computadorizada do segmento anterior (ACSA) para o diagnóstico de certeza de catarata branca intumescente (CBI), e com isto desenvolver uma estratégia cirúrgica que previna o aparecimento da capsulotomia descontínua, como o sinal da bandeira argentina ${ }^{(5)}$.

\section{Métodos}

Foram revistos os prontuários entre 2002 e 2007 de pacientes com catarata branca e nigra Os autores se basearam no critério de Osher ${ }^{(5)}$ e Fishkind ${ }^{(6)}$ quanto à avaliação biomicroscópica para classificá-los em catarata branca e nigra. Foram excluídos os que não apresentaram todos os dados como ângulo, densidade, espessura e forma do cristalino e ACD (profundidade de câmara anterior), fornecido pela análise computadorizada do segmento anterior.

Classificamos os olhos com catarata branca, em $\mathrm{CBN}$ - catarata branca normotensa e aquelas com espessura do cristalino $\geq 5.50 \mathrm{~mm}$, o ângulo da câmara anterior $<45^{\circ}$ e forma esferóide do cristalino como CBI - catarata branca intumescente ${ }^{(7)}$.

O critério laboratorial foi fornecido pelos índices da tomografia de segmento anterior como espessura e forma do cristalino, associado ao valor do ângulo camerular e volume da câmara anterior.

\section{Resultados}

São 22 olhos com catarata branca, 11 olhos com catarata nigra, conforme Tabelas 1 e 2.
Na Tabela 3, observa-se a espessura do cristalino. A forma do cristalino se observa na Tabela 4.

Os parâmetros analisados nas tabelas 5 e 6 não apresentaram diferenças significativas entre os dois grupos.

Na Tabela 7, podemos acompanhar os dados mais relevantes encontrados na CBI e CBN (Tabela 2), e analisar comparativamente.

Comparando-se os 22 olhos de pacientes com catarata branca e 11 olhos de pacientes com catarata nigra, por meio do teste t, apenas a variável espessura do cristalino apresenta diferença entre os dois grupos, com valor de $\mathrm{p}<0,001$. Para a variável densidade foi realizado o teste não paramétrico de Mann-Whitney (uma vez que essa variável não possui distribuição aproximadamente normal, pelo teste de Kolmogorov Smirnov), apresentando o valor de $p=0,001$, portanto existe diferença na variável densidade entre os grupos catarata branca e nigra (Tabela 8).

Comparando as médias das variáveis ângulo camerular, VCA e EC por meio do teste $t$ o valor de $p$ foi $>0,05$, portanto não existe diferença entre essas variáveis nos dois grupos, no entanto para a variável ACD o valor de $\mathrm{p}$ foi de 0,016 , portanto existe diferença na variável ACD entre os grupos de catarata branca normotensa e catarata branca intumescente (Tabela 9).

Para a variável densidade foi utilizado o teste não paramétrico Mann-Whitney (uma vez que a variável não apresentava distribuição aproximadamente normal pelo teste de Kolmogorov Smirnov). O valor de $\mathrm{p}$ foi 0,82 para o teste de Mann-Whitney, portanto não existe diferença entre as médias das densidades entre os dois tipos de catarata branca (Tabela 10).

\section{Discussão}

A catarata branca deve ser diagnosticada e classificada corretamente e assim permitir ao cirurgião recomendar a melhor estratégia cirúrgica. Sugerimos uma classificação, baseada no aspecto biomicroscópico associado à tomografia do segmento anterior.

À biomicroscopia do segmento anterior na lâmpada de fenda, o cristalino apresenta-se branco pérola, com midríase média, e com protrusão na câmara anterior, que está mais estreita que o normal. Esta

\begin{tabular}{ll}
\multicolumn{2}{c}{ Catarata branca } \\
\hline normotensa $(\mathrm{CBN})$ & intumescente $(\mathrm{CBI})$ \\
\hline - simples & \\
- morganiana & \\
\hline
\end{tabular}


Tabela 1

Grupo 1 - Catarata branca Análise computadorizada do segmento anterior

\begin{tabular}{|c|c|c|c|c|c|c|c|c|c|c|}
\hline Idade & AV & PIO & Ângulo & VCA & Dens & EC & For. Cris. & ACD & Ref. pós & AV pós \\
\hline 68 & PL & 12 & 58.0 & 194 & 69.0 & 5.07 & Esferiforme & 3.21 & $+0.50-1.25125^{\circ}$ & $20 / 30$ \\
\hline 68 & MM & 15 & 27.3 & 104 & 62.5 & 4.67 & Normal & 2.83 & +1.50 & $20 / 30$ \\
\hline 68 & MM & 15 & 38.8 & 133 & 62.5 & 4.67 & Normal & 4.32 & +1.50 & $20 / 30$ \\
\hline 67 & PL & & 37.4 & 223 & 91.0 & 4.27 & Esferiforme & 2.33 & $+0.50175^{\circ}$ & $20 / 400$ \\
\hline 69 & PL & 14 & 43.3 & 128 & 79.1 & 4.63 & Normal & 3.27 & & \\
\hline 69 & PL & 14 & 50.7 & 220 & 79.1 & 4.63 & Normal & 5.44 & & \\
\hline 58 & MM & 14 & 52.0 & 125 & 60.2 & 5.54 & Esferiforme & 2.18 & +1.0 & $20 / 20$ \\
\hline 62 & MM & & 38.9 & 221 & 100 & 5.36 & Normal & 3.06 & $-1.50-0.75180^{\circ}$ & $20 / 30$ \\
\hline 65 & MM & 17 & 34.4 & 141 & 100 & 5.47 & Normal & 2.26 & & $20 / 200$ \\
\hline 65 & MM & 17 & 48.8 & 160 & 100 & 5.47 & Normal & 2.26 & & $20 / 200$ \\
\hline 59 & PL & & 54.3 & 132 & 100 & 5.22 & Normal & 2.88 & $+1.0-0.5075^{\circ}$ & $20 / 20$ \\
\hline 59 & PL & & 43.9 & 196 & 100 & 5.22 & Normal & 4.62 & $+1.0-0.5075^{\circ}$ & $20 / 20$ \\
\hline 55 & PL & & 53.2 & 171 & 69 & 5.43 & Esferiforme & 2.77 & Plana & $20 / 30$ \\
\hline 42 & PL & 12 & 33.0 & 180 & 100 & 5.22 & Esferiforme & 2.73 & +1.0 & $20 / 20$ \\
\hline 76 & PL & & 34.0 & 137 & 100 & 4.79 & Normal & 3.01 & $-0.50130^{\circ}$ & $20 / 30$ \\
\hline 76 & PL & & 34.0 & 149 & 100 & 4.79 & Normal & 3.01 & $-0.50130^{\circ}$ & $20 / 30$ \\
\hline 77 & PL & & 39.1 & 85 & 100 & 4.81 & Esferiforme & 2.33 & $-0.5005^{\circ}$ & $20 / 40$ \\
\hline 77 & PL & & 44.0 & 125 & 100 & 4.81 & Esferiforme & 2.33 & $-0.5005^{\circ}$ & $20 / 40$ \\
\hline 78 & $20 / 400$ & 14 & 49.7 & 214 & 40.2 & 4.77 & Normal & 2.91 & $-1.0-1.0110^{\circ}$ & $20 / 30$ \\
\hline 74 & $20 / 400$ & & 29.7 & 199 & 100 & 5.80 & Esferiforme & 2.51 & $-2.50180^{\circ}$ & $20 / 30$ \\
\hline 62 & PL & 13 & 34.5 & 99 & 100 & 5.85 & Esferiforme & 2.34 & & \\
\hline 62 & PL & 13 & 34.5 & 201 & 100 & 5.85 & Esferiforme & 2.34 & & \\
\hline
\end{tabular}

$\mathrm{AV}=$ acuidade visual pela tabela de Snellen; $\mathrm{PIO}=$ pressão intra-ocular em mmHg; VCA = volume da câmara anterior em milímetros; Dens = densitometria do cristalino; EC = espessura do cristalino em milímetros; For. Cris. = forma do cristalino; ACD = profundidade da câmara anterior em milímetros; Ref. pós = refração pós-operatória e dioptria; AV pós = acuidade visual pós-operatória obtida pela tabela de Snellen

Tabela 2

Grupo 2 - Catarata nigra Análise computadorizada do segmento anterior

\begin{tabular}{ccccccccccc}
\hline Idade & AV & PIO & Ângulo & VCA & Dens & EC & For. Cris. & ACD & Ref. pós & AV pós \\
\hline 92 & PL & 14 & 28.0 & & & 4.84 & Normal & 3.26 & 26 & $20 / 400$ \\
74 & PL & 15 & 23.5 & 127 & 100 & 4.26 & Normal & 2.65 & $-0.7590^{\circ}$ & $20 / 200$ \\
81 & PL & 14 & 62.0 & 180 & 60.0 & 4.58 & Normal & 2.90 & $20 / 200$ \\
81 & PL & 14 & 62.0 & 170 & 60.0 & 4.58 & Normal & 4.43 & & $20 / 200$ \\
89 & $20 / 100$ & & 53.3 & 102 & 32.6 & 4.84 & Normal & 2.37 & $+0.50-1.0100^{\circ}$ & $20 / 50$ \\
70 & $20 / 100$ & 11 & 42.4 & 228 & 58.0 & 4.47 & Normal & 3.27 & -0.50 & $20 / 50$ \\
70 & $<20 / 400$ & & 18.9 & 155 & 82.2 & 4.70 & Normal & 2.81 & $+1.50-1.0115^{\circ}$ & $20 / 40$ \\
76 & $20 / 100$ & & 30.7 & 129 & 42.9 & 4.30 & Normal & 2.29 & $-1.0135^{\circ}$ & $20 / 30$ \\
76 & 20/100 & & 48.8 & 203 & 42.9 & 4.30 & Normal & 2.29 & $-1.0135^{\circ}$ & $20 / 30$ \\
80 & PL & 12 & 48.3 & 225 & 60.0 & 4.82 & Normal & 3.42 & -1.0 & $20 / 40$ \\
86 & 20/100 & 12 & 42.5 & 255 & 52.3 & 4.18 & Normal & 3.49 & $-1.25180^{\circ}$ & $20 / 30$ \\
\hline
\end{tabular}

$\mathrm{AV}=$ acuidade visual pela tabela de Snellen; PIO = pressão intra-ocular em mmHg; VCA = volume da câmara anterior em milímetros; Dens = densitometria do cristalino; EC = espessura do cristalino em milímetros; For. Cris. = forma do cristalino; ACD = profundidade da câmara anterior em milímetros; Ref. pós = refração pós-operatória e dioptria; AV pós = acuidade visual pós-operatória obtida pela tabela de Snellen 
Tabela 3

Espessura do cristalino em milímetros

\begin{tabular}{lcccccc}
\hline & CBI & Olhos & \% & CBN & Olhos & $\%$ \\
\hline Mínimo & $5,36 \mathrm{~mm}$ & $1 / 8$ & 12,50 & $4,27 \mathrm{~mm}$ & $1 / 7$ & 14,28 \\
Máximo & $6,35 \mathrm{~mm}$ & $2 / 8$ & 25,00 & $5,22 \mathrm{~mm}$ & $1 / 7$ & 14,28 \\
Média & $5,85 \mathrm{~mm}$ & $5 / 8$ & 62,50 & $4,92 \mathrm{~mm}$ & $5 / 7$ & 71,43 \\
\hline
\end{tabular}

$\mathrm{CBI}=$ catarata branca intumescente $\mathrm{CBN}=$ catarata branca normotensa
Tabela 4

Forma do cristalino

\begin{tabular}{lcccc}
\hline & CBI - \% & Olhos & CBN - \% & Olhos \\
\hline Esferiforme & 53,3 & 5 & - & -- \\
Normal & 47,7 & 3 & 100 & 7
\end{tabular}

$\mathrm{CBI}=$ catarata branca intumescente $; \mathrm{CBN}=$ catarata branca normotensa
Tabela 5

\section{Ângulo camerular}

\begin{tabular}{lcccccc}
\hline & CBI & Olhos & $\%$ & CBN & Olhos & $\%$ \\
\hline Mínimo & $32,5^{\circ}$ & $1 / 8$ & 12,50 & $30^{\circ}$ & $1 / 7$ & 14,28 \\
Máximo & $42,3^{\circ}$ & $1 / 8$ & 12,50 & $39^{\circ}$ & $1 / 7$ & 14,28 \\
Média & $36,9^{\circ}$ & $6 / 8$ & 75,00 & $36,35^{\circ}$ & $5 / 7$ & 71,43 \\
\hline
\end{tabular}

$\mathrm{CBI}=$ catarata branca intumescente $\mathrm{CBN}=$ catarata branca normotensa

\section{Tabela 6}

Volume da câmara anterior

\begin{tabular}{lcccccc}
\hline & CBI & Olhos & $\%$ & CBN & Olhos & $\%$ \\
\hline Mínimo & $85 \mathrm{~mm}^{3}$ & $1 / 8$ & 12,50 & $109 \mathrm{~mm}^{3}$ & $1 / 7$ & 14,28 \\
Máximo & $223 \mathrm{~mm}^{3}$ & $1 / 8$ & 12,50 & $220 \mathrm{~mm}^{3}$ & $1 / 7$ & 14,28 \\
Média & $159,68 \mathrm{~mm}^{3}$ & $6 / 8$ & 75,00 & $164,50 \mathrm{~mm}^{3}$ & $5 / 7$ & 71,43
\end{tabular}

$\overline{\mathrm{CBI}}=$ catarata branca intumescente $\mathrm{CBN}=$ catarata branca normotensa

Tabela 7

Comparação entre CBI e CBN

\begin{tabular}{lcc}
\hline & CBI & CBN \\
\hline Volume câmera anterior & $159,68 \mathrm{~mm}^{3}$ & $164,50 \mathrm{~mm}^{3}$ \\
Ângulo camerular & $32,5^{\circ}$ a $42,3^{\circ}$ & $30^{\circ}$ a $39^{\circ}$ \\
Espessura do cristalino & de 5.36 a $6.35 \mathrm{~mm}$ & de 4.27 a $5.22 \mathrm{~mm}$ \\
Densitometria & de $69 \%$ a $100 \%$ & de $62.5 \%$ a $100 \%$ \\
Forma do cristalino & esferiforme & normal \\
\hline
\end{tabular}

$\mathrm{CBI}=$ catarata branca intumescente $\mathrm{CBN}=$ catarata branca normotensa

Tabela 8

Comparando catarata branca e catarata nigra

\begin{tabular}{lccccccc}
\hline variáveis & $\begin{array}{c}\text { Número } \\
\text { de olhos }\end{array}$ & $\begin{array}{c}\text { Tipo } \\
\text { catarata }\end{array}$ & mínimo & máximo & média & $\begin{array}{c}\text { desvio } \\
\text { padrão }\end{array}$ & $\begin{array}{c}\text { Teste t } \\
\text { valor de p }\end{array}$ \\
\hline Ângulo & 22 & branca & 27,3 & 58,0 & 41,52 & 8,77 & 0,95 \\
camerular & 11 & nigra & 18,9 & 62,0 & 41,86 & 14,88 & \\
VCA & 22 & branca & 85 & 223 & 160,77 & 43,01 & 0,34 \\
& 10 & nigra & 102 & 255 & 177,40 & 50,15 & \\
EC & 22 & branca & 4,27 & 5,85 & 5,11 & 0,45 & $<0,001$ \\
& 11 & nigra & 4,18 & 4,84 & 4,53 & 0,25 & \\
ACD & 22 & branca & 2,18 & 5,44 & 2,95 & 0,84 & 0,83 \\
& 11 & nigra & 2,29 & 4,43 & 3,02 & 0,65 & \\
\hline
\end{tabular}




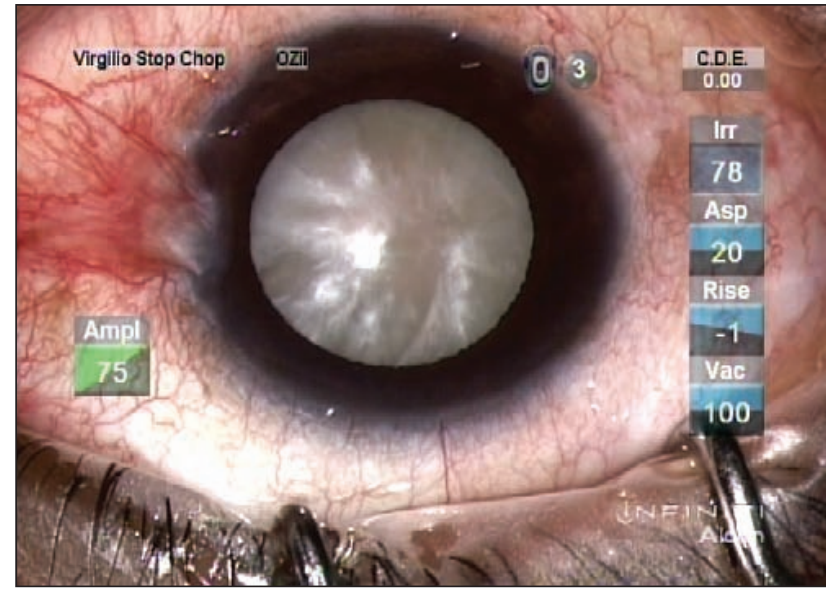

Figura 1: Aspecto clínico de CBI na biomicroscopia

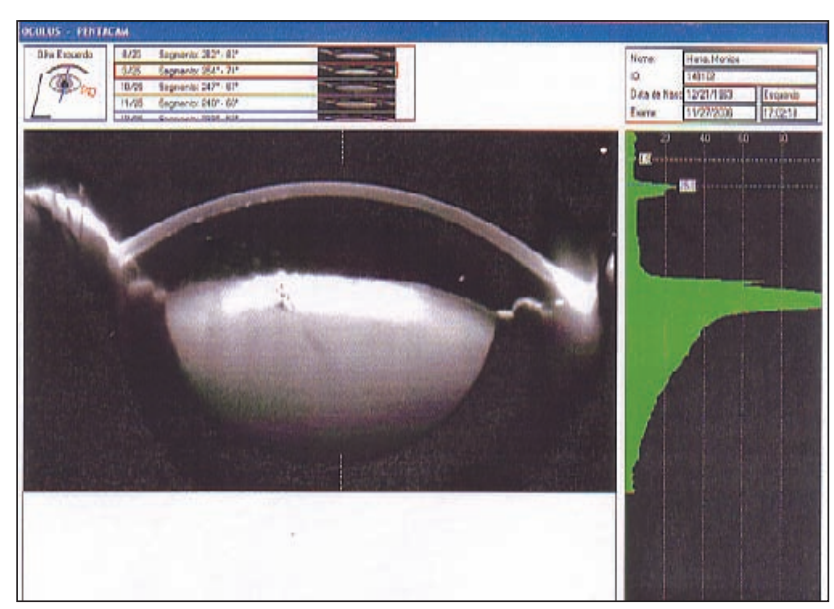

Figura 3: Tomografia do segmento anterior em CBN

imagem nos sugere que se trata de CBI (Figura 1).

Como propedêutica auxiliar, recomendamos a ultra-sonografia B para avaliar a possibilidade de outras doenças associadas, em especial da retina e do vítreo.

O exame conhecido como análise computadorizada do segmento anterior realizado com o tomógrafo de segmento anterior pelo sistema de Scheimpflug (Pentacam - Oculus ${ }^{\circledR}$ ) nos informa: a profundidade da câmara anterior de maneira topográfica, a densidade, a forma e as dimensões do cristalino, além de avaliar o ângulo camerular (Figuras 2, 3 e 4).

Numa CBN ou em uma morganiana, o maior problema no momento da cirurgia é visualizar a cápsula anterior para a realização da capsulorrexe; na CBI o desafio é visualizar e prevenir para que ela não se complique em uma capsulotomia descontínua, mais conhecida como sinal da bandeira Argentina, descrita por Perrone e Albertazzi no Video Film Festival da Americam

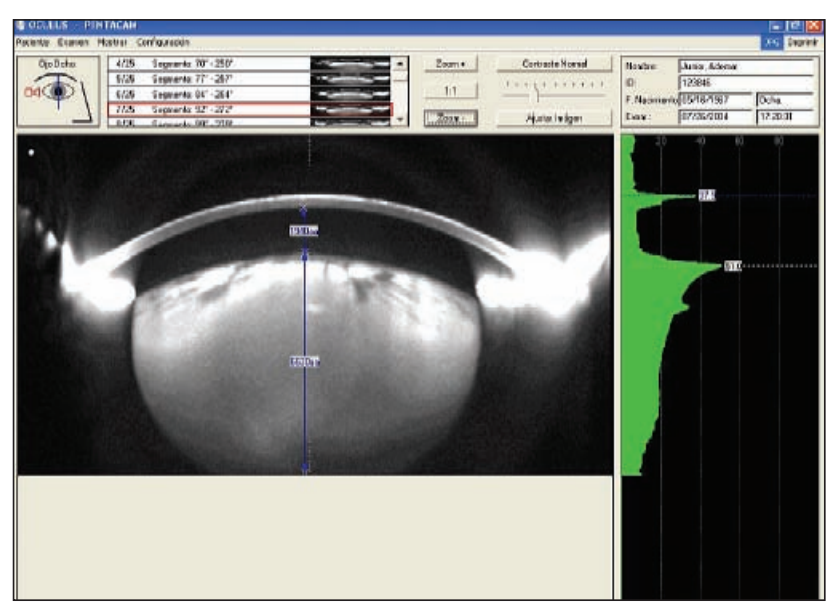

Figura 2: Tomografia do segmento anterior em CBI

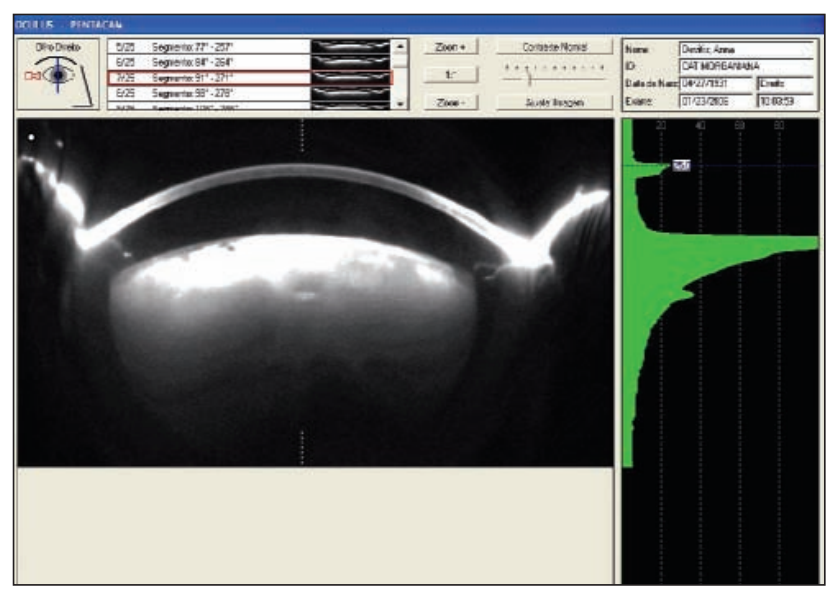

Figura 4: Tomografia do segmento anterior em CBN morganiana

Society of Cataract And Refractive Sugery, em $2000{ }^{(8)}$ (Figura 5).

No vídeo Competition do XXIV Congress of the European Society of Cataract and Refractive Sugery ${ }^{(7)}$ citamos algumas características que em nossa opinião ${ }^{(9)}$ seriam altamente sugestivas de CBI e que não encontram referência bibliográfica na literatura pesquisada. Elas são:

- Forma do cristalino: esferiforme, perdendo seu aspecto de lente biconvexa habitual quando vista de perfil;

- Espessura do cristalino: na linha média, maior que $5.50 \mathrm{~mm}$;

- Câmara anterior: profundidade $\leq 2.0 \mathrm{~mm}$;

- Ângulo camerular: $\leq 45^{\circ}$.

O presente trabalho nos evidencia de maneira muito clara que o fator mais importante no ACSA é a espessura do cristalino seguido da alteração da sua forma e acreditamos ser o primeiro relato na literatura. 
A densitometria pode ser de valor, pois uma densidade muito elevada poderia sugerir um núcleo muito denso, o que não é freqüente em CBI e sim em branca morganiana.

Em todos os pacientes houve melhora da acuidade visual, não havendo perda do número de linhas de visão. No grupo de catarata branca: 2 olhos apresentaram a melhor acuidade visual corrigida (MAVC) de 20/400 e 20/200 respectivamente, devido a alterações maculares. O grupo da catarata nigra apresentou MAVC de 20/400 em 2 olhos e 20/200 em 2 olhos devido a retinopatia diabética e DMRI respectivamente.

A previsibilidade biométrica no grupo de catarata branca mostrou 7 olhos com equivalente esférico de \pm 0,50 dioptria e 5 olhos com equivalente esférico $\pm 1,50$ dioptrias. A previsibilidade biométrica no grupo de catarata nigra mostrou-se adequada com todos os olhos com equivalente esférico entre $\pm 1,0$ dioptrias. Vide Tabela 1 e 2 .

Se não se tem o diagnóstico correto de CBI, ao iniciar a capsulotomia, ela poderá correr de maneira incontrolável em direção à periferia, estacionando geralmente ao nível da inserção da zônula.

Este mecanismo foi explicado por Assia ${ }^{(10)}$ da seguinte forma: devido ao aumento da pressão dentro do saco capsular logo após a punção, há saída do conteúdo

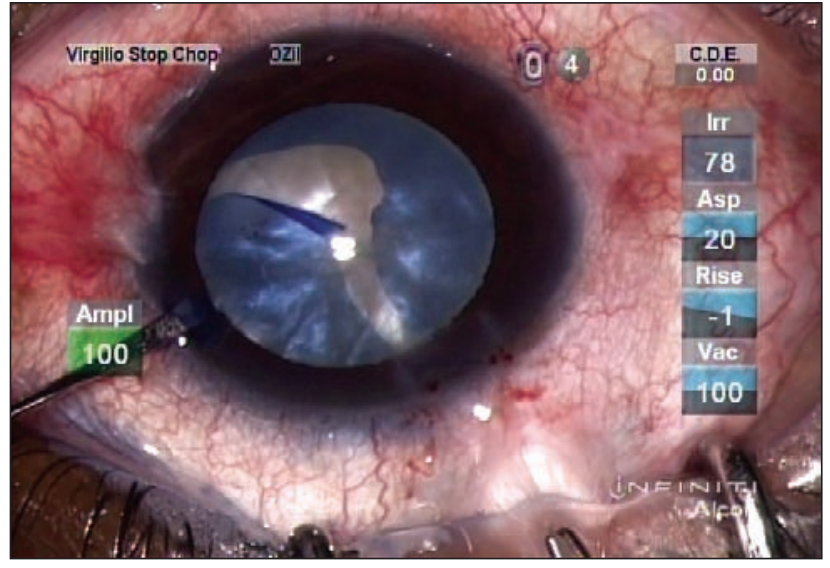

Figura 5: Sinal da bandeira Argentina

intra-sacular que inicialmente, com muita pressão, rasga a cápsula de maneira desordenada. À medida que se abre em direção à periferia, a pressão vai caindo e ao chegar a nível da inserção zonular estaciona, porque a pressão já não é suficiente e porque na periferia a cápsula tem o "reforço" da inserção de zônula. Esta inserção zonular foi classificada em dois tipos: os que se aderem na cápsula anterior de forma simples ou direta e aqueles que aderem em forma de leque.

De acordo com os autores deste trabalho, nesta situação, com a capsulotomia irregular e descontínua até

Tabela 9

\section{Cataratas brancas}

\begin{tabular}{lccccccc}
\hline Variáveis & Tipo catarata branca & $\begin{array}{c}\text { Número } \\
\text { olhos }\end{array}$ & Mínimo & Máximo & Média & $\begin{array}{c}\text { Desvio } \\
\text { padrão }\end{array}$ & $\begin{array}{c}\text { Teste t } \\
\text { Valor de } \boldsymbol{p}\end{array}$ \\
\hline Ângulo & normotensa & 12 & 27,3 & 54,3 & 41,51 & 8,29 & 0,99 \\
camerular & intumescente & 10 & 29,7 & 58,0 & 41,54 & 9,76 & \\
VCA & normotensa & 12 & 104 & 221 & 161,25 & 40,67 & 0,96 \\
& intumescente & 10 & 85 & 223 & 160,20 & 47,89 & \\
EC & normotensa & 12 & 4,63 & 5,47 & 4,97 & 0,34 & 0,14 \\
ACD & intumescente & 10 & 4,27 & 5,85 & 5,27 & 0,53 & \\
& normotensa & 12 & 2,26 & 5,44 & 3,32 & 0,97 & 0,016 \\
\hline
\end{tabular}

Tabela 10

\section{Densitometria comparativa}

\begin{tabular}{lccccccc}
\hline Variáveis & $\begin{array}{c}\text { Número } \\
\text { de olhos }\end{array}$ & $\begin{array}{c}\text { Tipo } \\
\text { catarata }\end{array}$ & Mínimo & Máximo & $\begin{array}{c}\text { Média } \\
\text { padrão }\end{array}$ & Desvio & Valor de p \\
\hline densitometria & 22 & branca & 40,2 & 100,0 & 86,94 & 18,38 & 0,001 \\
& 10 & nigra & 32,6 & 100,0 & 59,09 & 19,67 & \\
\hline
\end{tabular}


a periferia, o próximo passo é evitar que ela posteriorize, o que se consegue na maioria dos casos aspirando-se o conteúdo intra-sacular com a ponta do faco, utilizando parâmetros baixos incluindo a altura da garrafa de infusão. Se há núcleo duro, se luxa para câmara anterior e se realiza uma faco supracapsular, lenta, segura, com muito viscoelástico e a seguir o implante da LIO dentro do saco capsular e finaliza-se abrindo ou melhorando a capsulotomia anterior. Se não há possibilidade de continuar com faco, pode-se converter em extracapsular. Quando a capsulotomia se posterioriza com perda de núcleo em câmara vítrea, deve se realizar uma boa limpeza do vítreo e do material corticonuclear e deixar o paciente afácico e encaminhar ao retinólogo para o tratamento adequado.

\section{Conclusão}

Fazer o diagnóstico correto de CBI é fundamental, pois se trata de uma condição de alto risco de complicação per operatória. Este diagnóstico se faz no exame clínico e com a imagem da tomografia de segmento anterior e com os índices sugeridos por este trabalho, em especial uma espessura do cristalino maior de $5.36 \mathrm{~mm}$ e o aspecto esferiforme do mesmo. Outras publicações com maior número de casos poderão comprovar os nossos resultados.

\section{Abstract}

Purpose: To identify aspects of the image test to arrive at the sure diagnosis of intumescent white cataract. Methods: Two groups of eyes with white and nigra cataracts were studied using clinical and laboratorial criteria. Results: The width of the lens $\geq 5.36 \mathrm{~mm}$ and its spherical aspect are the two data that provided us with a sure diagnosis of intumescent white cataract. Conclusion: The use of the image test provides data for the sure diagnosis of intumescent white cataract.

Keywords: Cataract/diagnosis; Anterior eye segment; Image processing, computer-assisted; Image interpretation, computer-assisted

\section{REFERÊNCIAS}

1. Ermiss SS, Oztürk F, Inan UU. Comparing the efficacy and safety of phacoemulsification in white mature and other types of senile cataracts. Br J Ophthalmol. 2003; 87(11):1356-9.

2. Brazitikos PD, Tsinopoulos IT, Papadopoulos NT, Fotiadis K, Stangos NT. Ultrasonographic classification and phacoemulsification of white senile cataracts. Ophthalmology. 1999; 106(11):2178-83.
3. Chan DD, Ng AC, Leung CK, Tse RK. Continuous curvilinear capsulorhexis in intumescent or hypermature cataract with liquefied cortex. J Cataract Refract Surg. 2003; 29(3): 431-4.

4. Richards JC, Harrison DC. Preoperative neodymium: YAG anterior capsulotomy in intumescent cataract: preventing extension of the capsular tear to the lens periphery. J Cataract Refract Surg. 2003; 29(8):1630-1.

5. Osher RH, Osher JM. Confronting the White Cataract: Techniques for neutralizing increased endocapsular pressure and improving visualization can facilitate surgical management. Cataract Refract Surg Today [ Internet]. 2003; Nov/Dec.[cited 2007 Jan 16]. Available from: http:// www.crstodayarchive.com/03_archive/1103/13.html

6. Fishkind WJ, Samuelson TW, Masket S. The white cataract: How to manage a cortically mature nucleus. Cataract Refract Surg Today[ Internet] . 2005; July: 19-20 [cited 2007 Jan 16]. Available from: http://www.crstoday.com/PDF\%20Articles/ 0705/CRST0705_cs_phaco.pdf

7. Centurion V, Caballero JC, Jorge VK. Intumescent mature cataract. Third prize for special cases [Video competition]. In: XXIV Congress of The European Society of Cataract and Refractive Sugeons. ESCRS / ALCON 2006. London, England, September 2006.

8. ASCRS Film Festival Winners: Runners-up: Argentinean Flag Sign [Video]. Producer Daniel M. Perrone. 2000.

9. Centurion V, Caballero JC, Lacava AC, Leal EB. Catarata blanca intumescente. In: Lorente R, Mendicute J. Cirugia del cristalino. Sevilla. In press 2008.

10. Assia EI, Apple DJ, Tsai JC, Morgan RC. Mechanism of radial tear formation and extension after anterior capsulectomy. Ophthalmology. 1991; 98(4):432-7.

\section{ENDEREÇO PARA CORRESPONDÊNCIA: \\ Av. Ibirapuera, 624 - Ibirapuera \\ CEP 04028-000 - São Paulo - SP - Brasil \\ E-mail: centurion@imo.com.br}

